\title{
.
}

\section{Geometrical Modeling of Steel Ropes}

\author{
Eva Stanová \\ Technical University of Košice \\ Faculty of Civil Engineering, Institute of Construction Technology and Management \\ e-mail:eva.stanova@tuke.sk
}

\begin{abstract}
The paper deals with the mathematical geometric modeling of the ropes of circular cross- section. Such rope can be formed from strands of different shapes. There is considered steel rope made up of six strands, whose crosssection has oval, triangular or circular profil in this paper. The wires of these types of the strands are presented by parametric equations of the wire axis. The equations are implemented in the Pro/Engineer Wildfire V5 software for creating the geometrical model of the strand.
\end{abstract}

Key words: wire rope, strand of a rope, geometrical model

\section{Introduction}

Wire ropes are elements of machine engineering but their use is much more extensive. They are mainly used in engineering, mining and construction._Basic structural component of them is a steel wire, which can have different profile shapes [1]. Steel wires form a strand of a rope. They also can have different shapes. The individual strands ultimately create the rope.

Many authors have dealt with the design and creation of the geometric model of the strands and ropes. Some types of circular [2,3], triangular [5, 6] and oval strands [4] are described.

In this paper, one of the ways to express mathematically a circular rope made from one of three types of the strands is described and its use for creating the geometric model by implementation to the computer-aided design software ProEngineer Wildfire is demonstrated. The geometric model can be used for computer simulation and subsequent analysis of the wire ropes.

\section{Basic assumptions and problem formulation}

We will consider steel rope of circular profile made up of six strands, whose cross-section can be: 
- oval,

- triangular,

- circular.

The strands are made of round wires, which are wound on the core strand in one or two layers. The core of the strand according to its shape composed of one or more wires. The strands are subsequently helically laid around the rope core. Each wire of the layer strand is therefore a double wound and each wire of the strand core is simply wound. Mathematically, we express mathematically the axis of wires in a strand. This allows us to create a geometric model of the rope. Mathematical expressions will be in the form of parametric equations of axes curves of the individual wires. We will proceed as follows:

- we mathematically express the wires axes curves in the strands of mentioned cross sections,

- we express the mathematical formulation of the strand windings at the rope core,

- Based on this formulation we create geometric model of the rope.

\section{Mathematical expression of the wire axes in the strand layer}

The considered strand is made of steel wires, which are helically wound around the strand core in one or two layers._Denote $n_{0}$ the number of core wires, $\delta_{0}$ their diameter and $\Delta_{0}$ the gap between the wires. The _strand layer $j$ consists of $n_{j}$ wires with a diameter $\delta_{j}$ between which there is a gap $\Delta_{j} .{ }_{-}$We assume that there are not gaps between wires of neighboring layers. The angle of wire winding in one layer is $\alpha_{j}$.

\subsection{Oval strand}

The strand core consisst of $n_{0}$ straight wires arranged so that their axes points lie on one straight line._Points of wire axes of the first layer then lie partly on the line and partly on the circular curves (Fig. 1).

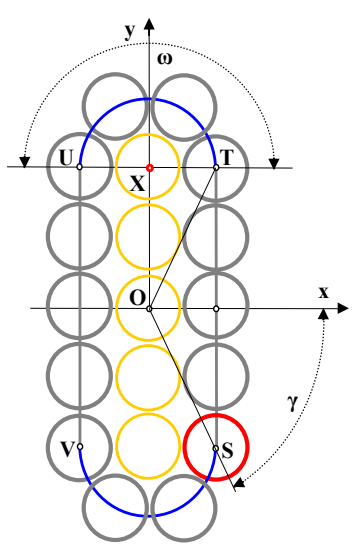

Figure 1: Cross-section of the $1^{\text {st }}$ layer

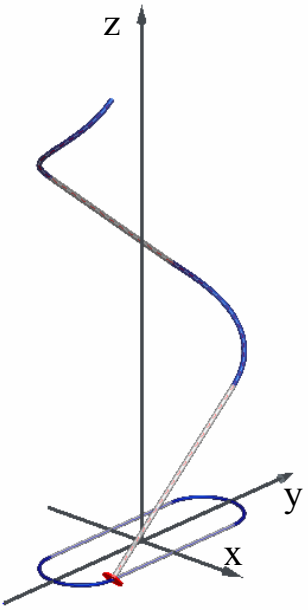

Figure 4: The centreline of the wire 
Let the right-hand Cartesian coordinate system $(O ; x, y, z)$ be placed so that the axis $z$ is identical with the axis $o_{S}$ of the strand. The curve of the wire axis consists of $k$ straight line segments and $k$ helix segments (Fig. 2). The parametric equations of the first line segment of the wire in the layer $j$ according to [6] have the form

$$
\begin{gathered}
x_{l_{j}}(\psi)=\frac{b_{j}}{2}, \\
y_{l_{j}}(\psi)=\frac{b_{j}}{2} \tan \left(\psi-\gamma_{j}\right), \\
z_{l_{j}}(\psi)=\frac{a+b_{j} \tan \left(\psi-\gamma_{j}\right)}{2 \tan \alpha_{j}},
\end{gathered}
$$

where $\psi \in\left\langle 0 ; 2 \gamma_{j}\right\rangle$ and

$$
\begin{gathered}
\gamma_{j}=\arctan \frac{a}{b_{j}}, \\
a=\left(n_{0}-1\right)\left(\delta_{0}+\Delta_{0}\right), \\
b_{j}=\delta_{0}+2(j-1) \delta_{j-1}+\delta_{j} .
\end{gathered}
$$

The equations of the first helix part are obtained as

$$
\begin{gathered}
x_{h_{j}}(\psi)=\frac{b_{j}}{2} \cos (\psi), \\
y_{h_{j}}(\psi)=\frac{a+b_{j} \sin (\psi)}{2}, \\
z_{h_{j}}(\psi)=\frac{2 a+b_{j} \psi}{2 \tan \alpha_{j}},
\end{gathered}
$$

where $\psi,\langle 0 ; \pi\rangle$ is the angle of rotation around the axis $o_{h}$.

The line and helix segments are repeated in the wire axis. Each of them is rotated by the angle $\kappa=(k-1) \pi$ and shifted of the length

$$
h_{\Delta_{j}}=\frac{b_{j} \pi+2 a}{2 \tan \alpha_{j}}
$$

relative to the axis $z$. The wires of one layer create the same surfaces. Therefore, each wire in the layer is given by the previous one shifted by a particular size $h_{w_{j}}$ in the axial direction. It can be calculated from the relationship

$$
h_{w_{j}}=\frac{h_{\Delta_{j}}}{n_{j}}
$$


So, the curve of axis of the single-helical wire $i$ in the layer $j$ can be expressed by parametric equations

$$
\begin{gathered}
x_{s}(\psi)=x_{w}(\psi) \cos \kappa-y_{w}(\psi) \sin \kappa \\
y_{s}(\psi)=x_{w}(\psi) \sin \kappa+y_{w}(\psi) \cos \kappa \\
z_{s}(\psi)=z_{w}(\psi)+(k-1) h_{\Delta_{j}}-(i-1) h_{w_{j}}
\end{gathered}
$$

in which we use the equations (1) - (3), $\psi \in\left\langle 0 ; 2 \gamma_{j}\right\rangle$ for line segment $(w=l)$ and the equations (7) - (9), $\psi \in\langle 0 ; \pi\rangle$ for helical segment $(w=h)$.

\subsection{Triangular strand}

Assume that the triangular strand have in the first layer $3 n_{1}$ wires. The points of wire axis lie on an equilateral triangle $\mathrm{ABC}$ in the section of the strand. Let the right-hand Cartesian coordinate system $(O ; x, y, z)$ be placed so that the $z$-axis is identical with the axis $o_{s}$ of the strand and the $x$-axis is perpendicular to the side $A B$ of the triangle $A B C$ (Fig. 3)._Like in the case of oval strand, the wire axis curve is formed by parts of the line and the cylindrical helix [5] that are repeated rotated by an angle $\kappa=(k-1) \frac{2 \pi}{3}$ and moved on size $(k-1) h_{\Delta j}$ (Fig. 4).
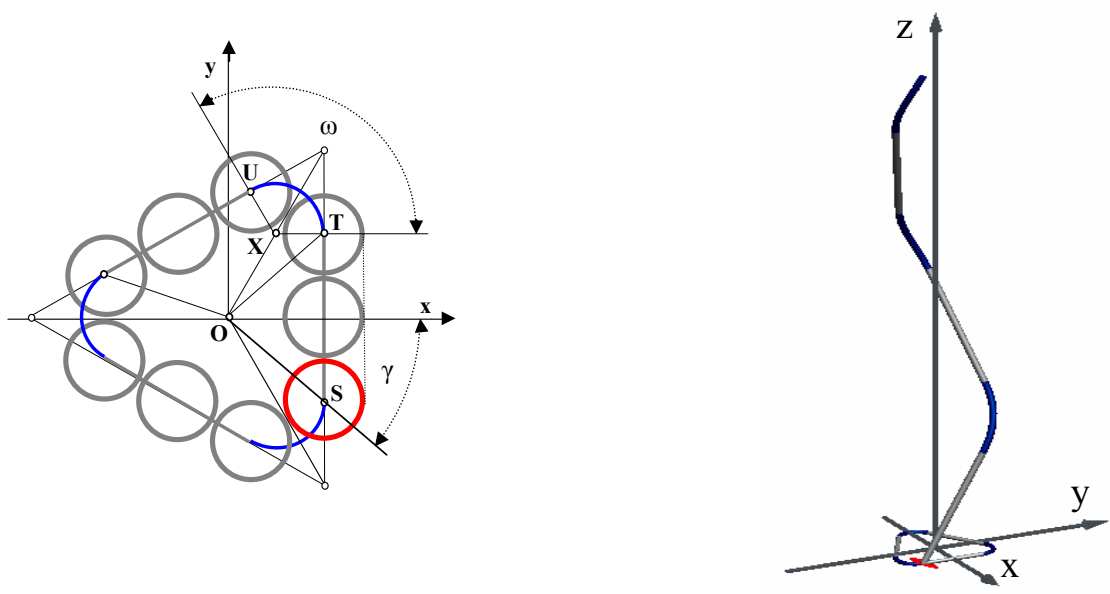

Figure 3: Cross-section of the $1^{\text {st }}$ layer

Figure 4: The centreline of the wire

The parametric equations of the line segment of the wire in the layer $j$ according to [6] have the form

$$
\begin{gathered}
x_{l j}(\psi)=\frac{c\left(n_{1}+1\right)+(j-1)\left(\delta_{1}+\delta_{2}\right)}{2} \\
y_{l_{j}}(\psi)=\frac{c\left(n_{1}+1\right)+(j-1)\left(\delta_{1}+\delta_{2}\right)}{2} \tan \left(\psi-\gamma_{j}\right)
\end{gathered}
$$




$$
z_{l j}(\psi)=\left[\frac{c\left(n_{1}+1\right)+(j-1)\left(\delta_{1}+\delta_{2}\right)}{2} \tan \left(\psi-\gamma_{j}\right)+\frac{\sqrt{3} a\left(n_{1}+1\right)}{2}\right] \cot \alpha_{j}
$$

where

$$
\begin{gathered}
\gamma_{j}=\arctan \frac{\left(n_{1}+1\right)\left(\delta_{1}+\Delta_{1}\right)}{c\left(n_{1}+1\right)+(j-1)\left(\delta_{1}+\delta_{2}\right)} \\
c=\frac{\left(\delta_{1}+\Delta_{1}\right)}{\sqrt{3}}
\end{gathered}
$$

and $\psi \in\left\langle 0 ; 2 \gamma_{j}\right\rangle$.

For the helix segment

$$
\begin{gathered}
x_{h j}(\psi)=\frac{c\left(n_{1}+1\right)}{2}+c\left[+(j-1) \frac{\delta_{1}+\delta_{2}}{2}\right] \cos \psi, \\
y_{h j}(\psi)=\frac{\sqrt{3} c\left(n_{1}+1\right)}{2}+\left[c+(j-1) \frac{\delta_{1}+\delta_{2}}{2}\right] \sin \psi, \\
z_{h j}(\psi)=\left\{\sqrt{3} c\left(n_{1}-1\right)+\left[c+(j-1) \frac{\delta_{1}+\delta_{2}}{2}\right] \psi\right\} \cot \alpha_{j},
\end{gathered}
$$

where $\psi,\left\langle 0 ; \frac{2 \pi}{3}\right\rangle$. Of these equations for the size of the displacement results the relationship

$$
h_{\Delta j}=\left\{\sqrt{3} c\left(n_{1}-1\right)+\left[c+(j-1) \frac{\delta_{1}+\delta_{2}}{2}\right] \frac{2 \pi}{3}\right\} \cot \alpha_{j} .
$$

Any curve of axis of the single-helical wire $i$ in the layer $j$ of triangular strand can be expressed by parametric equations (11) - (14) in which we use the equations (15) - (17), $\psi \in\left\langle 0 ; 2 \gamma_{j}\right\rangle$ for line segment $(w=l)$ and the equations (20)- (22), $\psi \in\left\langle 0 ; \frac{2 \pi}{3}\right\rangle$ for helical segment $(w=h)$.For the size of shift we use the equation (23).

\subsection{Circular strand}

We can derive parametric equations of the circular strand on the same principle as it is in the previous two types of strands. Assume that the triangular strand have in $j$ layer $n_{j}$ wires. Points of wire axes of one layer lie on a circle. The right-hand Cartesian coordinate system $(O ; x, y, z)$ is located in the same way as in the previous cases.

The centreline of the wire in the layer $j$ is defined by the parametric equations 


$$
\begin{gathered}
x_{s}(\psi)=r_{j} \cos \psi \\
y_{s}(\psi)=r_{j} \sin \psi \\
z_{s}(\psi)=r_{j} \psi \cot \alpha_{j}-(i-1) h_{w_{j}}
\end{gathered}
$$

where $\psi \in\langle 0 ; 2 \pi\rangle$ for one pitch lenght, $i=1,2, \ldots, n_{j}$ and

$$
h_{\Delta_{j}}=2 r_{j} \pi \cot \alpha_{j}
$$

is the length of the shift for other pitch lenghts.

\section{Mathematical expression of the wire axes in the rope}

Wire rope is formed by the strands helically laid around the core. Let us assume that $R_{s}$ is the lay radius and $\beta$ the lay angle of the strand in the rope. To obtain the mathematical expression of wire centreline in the rope we can use the coordinate system transformation [7]. The coordinate system $(O ; x, y, z)$ used in single helical laid wire we transform to coordinate system $\left(O^{\prime} ; x^{\prime}, y^{\prime}, z^{\prime}\right)$ where the axis $z^{\prime}$ is identical with the rope axis as is shown in Fig. 7.

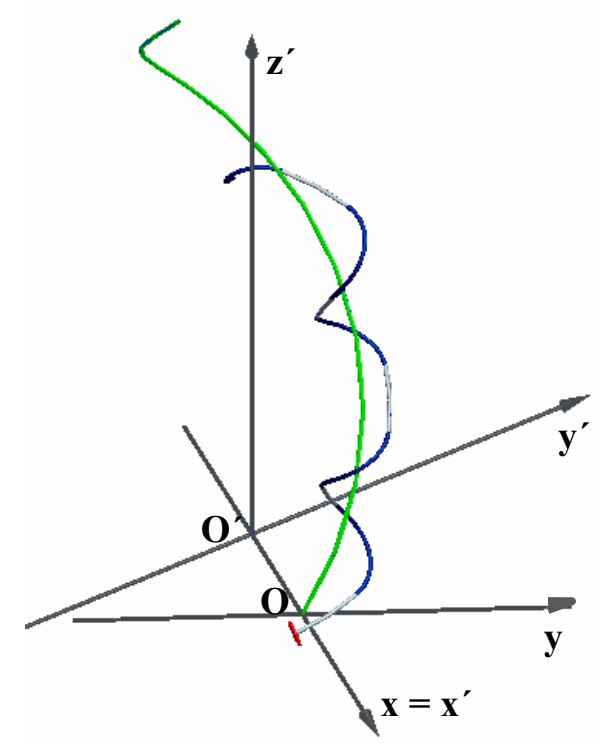

Figure 7: Transformation of the coordinate system

By using the above transformation, the following equations of the centreline of the wire $i$ in the layer $j$ are obtained:

$$
\begin{gathered}
x^{\prime}(\xi)=x_{s} \cos \left(\xi-\xi_{\Delta}\right)-y_{s} \sin \left(\xi-\xi_{\Delta}\right) \cos \beta+R \cos \left(\xi-\xi_{\Delta}\right), \\
y^{\prime}(\xi)=x_{s} \sin \left(\xi-\xi_{\Delta}\right)-y_{s} \cos \left(\xi-\xi_{\Delta}\right) \cos \beta+R \sin \left(\xi-\xi_{\Delta}\right),
\end{gathered}
$$




$$
z^{\prime}(\xi)=-y_{s} \sin \beta+R\left(\xi-\xi_{\Delta}\right) \cot \beta
$$

where $x_{s}, y_{s}$ are parametric equations of the single-helical wire axis. The angle $\xi \in\langle 0,2 \pi\rangle$ for one pitch length of the strand. Using parametric equations (28) - (30), it is necessary to express the angle $\psi$ by the angle $\xi$ in the equations of different types of strands.

There are models of the circular ropes formed of oval strands (Fig. 8a), triangular strands (Fig. 8b) and round strands (Fig. 8c) constructed for verify the correctness of the derived equations. Geometrical models are created by implementation of given parametric equations to the design software Pro/ENGINEER Wildfire v5 [8,9].
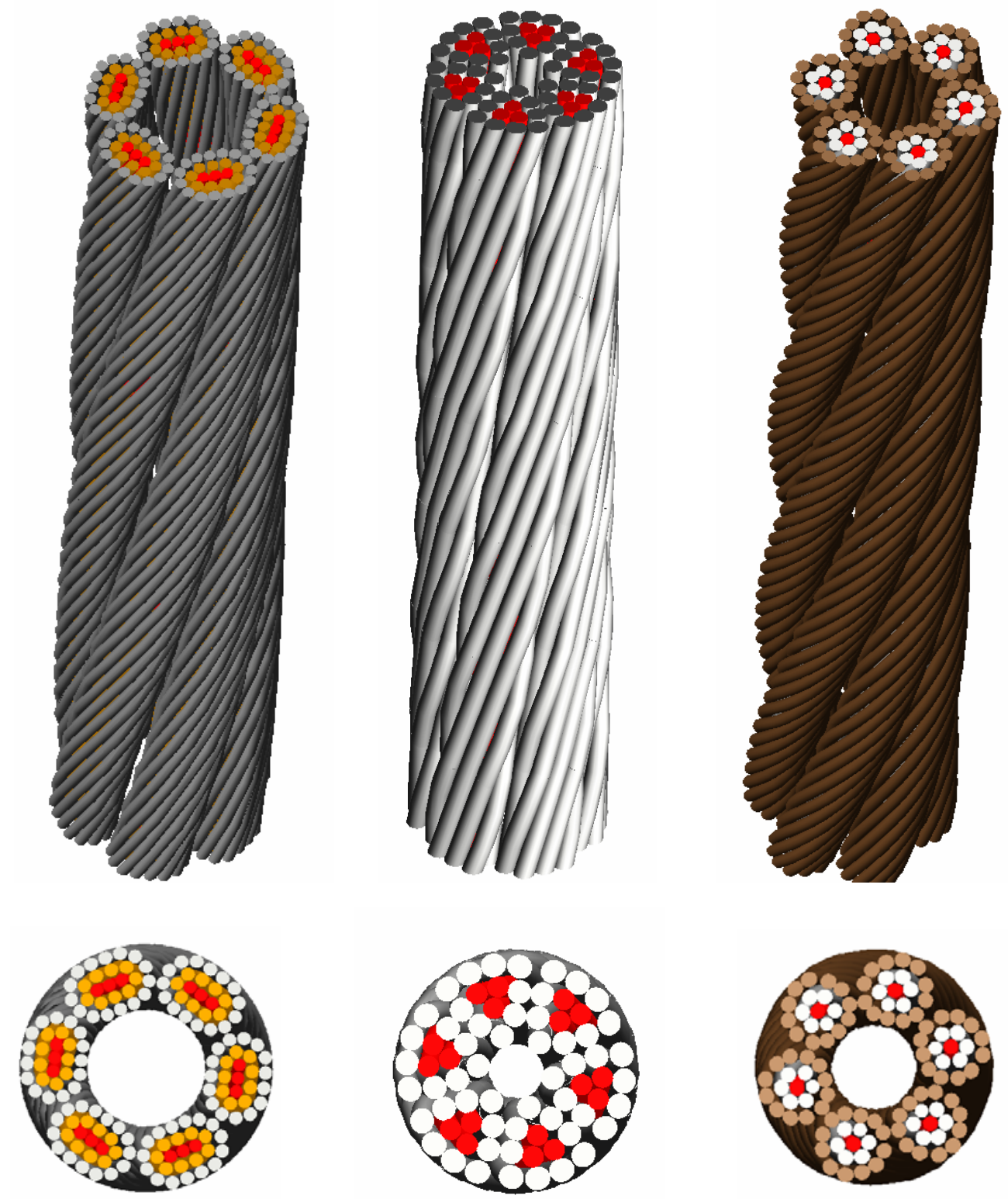

Figure 8: Model of the circular rope formed of: a) oval strands, b) triangular strands c) round strands 


\section{Conclusion}

The paper describes one of the possibilities of geometric modeling of circular ropes. It deals with the ropes created by the oval, triangular and round strands. There is mathematical expression in the form of parametric equations of individual simply wound wires in the mentioned types of strands listed. The parametric equations have variable parameters determining the number of wires in the layers. It allows the modeling of different types of the ropes.

For illustration, the geometric models of three ropes are created.

\section{Acknowledgements}

This work is a part of Research Project VEGA 1/0321/12: Theoretical and experimental analysis of adaptive rope and tensegrity systems under static and dynamic stress with considering the effects of wind and seismic.

\section{References}

[1] Feyer, K. (2007). Wire Ropes.Tension, Endurance, Reliability. Germany: Springer Verlag Berlin Heidelberg.

[2] Usabiaga, H., Pagalday, J. M. (2008). Analytical procedure for modeling recursively and wire by wire stranded ropes subjected to traction and torsion loads. International Journal of Solids and Structures, 45(21),.5503-20

[3] Stanová E, Fedorko G, Fabian M, Kmet S. (2011). Computer modelling of wire strands and ropes Part I: Theory and computer implementation. Advances in Engineering Software, 42(6), 305-15.

[4] Stanová E (2012). Geometry and Modeling of Oval Strand of $\mathrm{n}_{0}+\left(2 \mathrm{n}_{0}+4\right)+\mathrm{n}_{2}$ Type. SSP - Journal of Civil Engineering, Selected Scientific Papers, 7(2), 33-40. DOI: 10.2478/v10299-012-0004-3

[5] Stanová E (2008). Mathematical Expression of the Wire Axis in Trihedral Strand of Steel Rope. The International Journal of Transport \& Logistics,.8(14), 40 - 46

[6] Song J, Cao G, Cao Y, Wu R. (2012). Modeling method and analysis of geometric characteristic for the triangular strand rope. Modern Manufacturing Engineering; No.11, 1-7.

[7] Stanová E. (2013). Geometric Model of the Rope Created of Oval Strands. The International Journal of Transport \& Logistics, 13(26), 1-7. Retrieved February, 2013, on the web: http://www.sjf.tuke.sk/transportlogistics/?cat=18

[8] Fedorko, G., Molnár, V., Madáč, K. (2008). Fundamentals of application Pro / Engineer in technical design. Košice: Publishing of Faculty of Mining, Ecology, Process Control and Geotechnology, Technical University of Košice.

[9] Fabian, M., Spišák, E. (2009). Design and manufacture using CA .. technology. Brno : CCB. 